\title{
Characteristics Weak Galerkin Finite Element Methods for Convection-Dominated Diffusion Problems
}

\author{
Ailing Zhu, Qiang Xu, and Ziwen Jiang \\ School of Mathematical Sciences, Shandong Normal University, Jinan 250014, China \\ Correspondence should be addressed to Ziwen Jiang; ziwenjiang@163.com \\ Received 14 March 2014; Accepted 12 May 2014; Published 27 May 2014 \\ Academic Editor: Xinan Hao
}

Copyright (C) 2014 Ailing Zhu et al. This is an open access article distributed under the Creative Commons Attribution License, which permits unrestricted use, distribution, and reproduction in any medium, provided the original work is properly cited.

The weak Galerkin finite element method is combined with the method of characteristics to treat the convection-diffusion problems on the triangular mesh. The optimal order error estimates in $H^{1}$ and $L^{2}$ norms are derived for the corresponding characteristics weak Galerkin finite element procedure. Numerical tests are performed and reported.

\section{Introduction}

We will consider combining the method of characteristics with weak Galerkin finite element techniques to treat the model problem given by

$$
\begin{gathered}
a \frac{\partial u}{\partial t}+B \cdot \nabla u-\nabla \cdot(D \nabla u)=f, \quad \text { in } \Omega, t \in(0, T], \\
u(x, y, 0)=u_{0}(x, y), \quad(x, y) \in \Omega, \\
u(x, y, t)=\varphi(x, y, t), \quad(x, y) \in \partial \Omega, t \in(0, T],
\end{gathered}
$$

where $\Omega \subset \mathbf{R}^{2}$ is a bounded convex polygonal domain with the boundary $\partial \Omega, u$ is an unknown function, $a(x, y, t)$, $u_{0}(x, y)$, and $f(x, y, t)$ are known functions, $B(x, y, t)=$ $\left(b_{i}(x, y, t)\right)_{2 \times 1}$ is known bounded vector-valued functions, and $D=\left(d_{i, j}(x, y, t)\right)_{2 \times 2}$ is a symmetric, bounded matrix function. Assuming that the matrix $D$ satisfies the following condition, there exist positive constants $\alpha$, such that

$$
\alpha \xi^{\top} \xi \leq \xi^{\top} D \xi, \quad \forall \xi=\left(\xi_{1}, \xi_{2}\right) \in \Omega .
$$

For periodic problems we do away with boundary value condition $u(x, y, t)=\varphi(x, y, t),(x, y) \in \partial \Omega, t \in(0, T]$.

Here and in what follows, we will not write the independent $x, y, t$ for any functions unless it is necessary.

Let

$$
\psi(x, y, t)=\left[a(x, y, t)^{2}+b_{1}(x, y, t)^{2}+b_{2}(x, y, t)^{2}\right]^{1 / 2}
$$

and let the characteristics direction associated with the operator $a u_{t}+B \cdot \nabla u$ be denoted by $\tau=\tau(x, y, t)$, where

$$
\frac{\partial}{\partial \tau}=\frac{a}{\psi} \frac{\partial}{\partial t}+\frac{1}{\psi} B \cdot \nabla
$$

Then (1) can be put in the form

$$
\psi \frac{\partial u}{\partial \tau}-\nabla \cdot(D \nabla u)=f, \quad(x, y) \in \Omega, t \in(0, T] .
$$

The weak form for (1) seeks $u \in H^{1}(\Omega)$ such that $u(0)=$ $u_{0}$, and

$$
\left(\psi \frac{\partial u}{\partial \tau}, v\right)+(D \nabla u, \nabla v)=(f, v), \quad \forall v \in H_{0}^{1}(\Omega), t \in(0, T]
$$

Let $N>0$ be a positive integer, $t^{i}=i \Delta t(0 \leq i \leq N)$, and $\Delta t=T / N$. The characteristics derivative will be approximated basically in the following manner. Let

$$
\begin{aligned}
& \bar{x}=x-\frac{b_{1} \Delta t}{a}, \\
& \bar{y}=y-\frac{b_{2} \Delta t}{a},
\end{aligned}
$$


and note that

$$
\begin{aligned}
\psi \frac{\partial u}{\partial \tau} & \approx \psi^{i} \frac{u\left(x, y, t^{i}\right)-u\left(\bar{x}, \bar{y}, t^{i-1}\right)}{\left[(x-\bar{x})^{2}+(y-\bar{y})^{2}+(\Delta t)^{2}\right]^{1 / 2}} \\
& =a^{i} \frac{u\left(x, y, t^{i}\right)-u\left(\bar{x}, \bar{y}, t^{i-1}\right)}{\Delta t} .
\end{aligned}
$$

We use standard definitions for the Sobolev spaces $H^{s}(D)$ and their associated inner products $(\cdot, \cdot)_{s, D}$, norms $\|\cdot\|_{s, D}$, and seminorms $|\cdot|_{s, D}$ for $s \geq 0$. For example, for any integer $s \geq 0$, the seminorm $|\cdot|_{s, D}$ is given by

$$
|v|_{s, D}=\left(\sum_{|\alpha|=s} \int_{D}\left|\partial^{\alpha} v\right|^{2} d D\right)^{1 / 2},
$$

with the usual notation

$$
\begin{aligned}
\alpha & =\left(\alpha_{1}, \alpha_{2}\right), \\
|\alpha| & =\alpha_{1}+\alpha_{2}, \\
\partial^{\alpha} & =\partial_{x_{1}}^{\alpha_{1}} \partial_{x_{2}}^{\alpha_{2}} .
\end{aligned}
$$

The Sobolev norm $\|\cdot\|_{s, D}$ is given by

$$
\|v\|_{s, D}=\left(\sum_{j=0}^{s}|v|_{j, D}^{2}\right)^{1 / 2} .
$$

We use $\|\cdot\|$ for the $L^{2}$ norm.

Let $\chi$ be a normed space with the norm $\|\cdot\|_{\chi} \cdot L^{q}(0, T ; \chi)$ denote the space of the maps of $[0, T]$ into $\chi$ and define the following norms for $1 \leq q<\infty$ and suitable functions $v$ : $[0, T] \rightarrow \chi:$

$$
\|v\|_{L^{q}(0, T ; \chi)}=\left(\int_{0}^{T}\|v(t)\|_{\chi}^{q} d t\right)^{1 / q} .
$$

For $q=\infty$, the usual modification is made.

In many diffusion processes arising in physical problems, convection essentially dominates diffusion, and it is natural to seek numerical methods for such problems that reflect their almost hyperbolic nature. Convection-diffusion problems have been treated by various numerical methods [1-16]. Some methods of them treating convection-dominated diffusion problems exhibit stability limitation. The goal of this paper is to combine the method of characteristics with the weak Galerkin finite element method [17, 18] for convectiondominated diffusion equation. The principal gains from these procedures appear in time truncation. Approximation of $\partial u / \partial t$ by standard backward differencing leads to errors of the form $C \partial^{2} u / \partial t^{2} \Delta t$ in suitable norms, while characteristics method will be shown to yield $C \partial^{2} u / \partial t^{2} \Delta t$. In problems with significant convection, the solution changes much less rapidly in the characteristics $\tau$ direction than the $t$ direction in [19]. Thus, these characteristics schemes will permit the use of larger time steps, with corresponding improvements in efficiency, at no cost in accuracy. We will see that there is no stability limitation on the size of $\Delta t$.

An outline of the remainder of this paper is as follows. In Section 2, the characteristics weak Galerkin finite element scheme for the convection-diffusion initial boundary value problem (1) will be formulated. A generalized weak Galerkin elliptic projection is defined in Section 3. Optimal error estimations in both $H^{1}$ and $L^{2}$ norms of characteristics weak Galerkin finite element solution are proved in Section 4. The paper is concluded with some numerical experiments to illustrate the theoretical analysis in Section 5.

\section{Characteristics Weak Galerkin Finite Element Formulations}

In this section, we design the characteristics weak Galerkin finite element schemes for the initial boundary value problem (1). We consider the space of discrete weak functions and the discrete weak operator introduced in [17]. Let $\mathscr{T}_{h}=\{K\}$ be a triangulation partition of the domain $\Omega$ with mesh-size $h$. As usual, we assume the triangles $K$ to be shape-regular. For each element $K \in \mathscr{T}_{h}$, denote by $K^{0}$ and $\partial K$ the interior and the boundary of $K$, respectively. The boundary $\partial K$ consists of several "sides," which are edges. Denote by $\mathscr{F}_{h}$ the collection of all edges in $\mathscr{T}_{h}$. On each $K \in \mathscr{T}_{h}$, let $P_{j}\left(K^{0}\right)$ be the set of polynomials on $K^{0}$ with degree less than or equal to $j$. Likewise, on each $\partial K \in \mathscr{F}_{h}$, let $P_{j+1}(\partial K)$ be the set of polynomials on $\partial K$ with degree no more than $j+1$ (i.e., polynomials of degree $j+1$ on each line segment of $\partial K$ ).

A weak function on the region $K$ refers to a vector-valued function $v=\left\{v_{0}, v_{b}\right\}$ such that $v_{0} \in P_{j}\left(K^{0}\right)$ and $v_{b} \in P_{j+1}(\partial K)$ with $j \geq 0$. The first component $v_{0}$ can be understood as the value of $v$ in the interior of $K$, and the second component $v_{b}$ is the value of $v$ on the boundary of $K$.

Denote this space by $W(K, j)$

$$
W(K, j):=\left\{v=\left\{v_{0}, v_{b}\right\}: v_{0} \in P_{j}\left(K^{0}\right), v_{b} \in P_{j+1}(\partial K)\right\} .
$$

The corresponding finite element space would be defined by patching $W(K, j)$ over all the triangles $K \in \mathscr{T}_{h}$. In other words, the weak finite element space is given by

$$
S_{h}(j):=\left\{v=\left\{v_{0}, v_{b}\right\}:\left.\left\{v_{0}, v_{b}\right\}\right|_{k} \in W(K, j), \forall K \in \mathscr{T}_{h}\right\} .
$$

Denote by $S_{h}^{0}(j)$ the subspace of $S_{h}(j)$ with vanishing boundary values on $\partial \Omega$; that is,

$$
S_{h}^{0}(j):=\left\{v=\left\{v_{0}, v_{b}\right\} \in S_{h}(j),\left.v_{b}\right|_{\partial K \cap \partial \Omega}=0, \forall K \in \mathscr{T}_{h}\right\} .
$$

Let $V(K, j+1) \subset\left[P_{j+1}(K)\right]^{2}$ be a subspace of the set of vector-valued polynomials of degree no more than $j+1$ on $K$ and $\sum_{h}=\left\{\mathbf{q} \in\left(L^{2}(\Omega)\right)^{2}:\left.\mathbf{q}\right|_{K} \in V(K, j+1), \forall K \in \mathscr{T}_{h}\right\}$. 
For each $v=\left\{v_{0}, v_{b}\right\} \in S_{h}(j)$, the discrete weak gradient $\nabla_{d} v \in$ $\sum_{h}$ of $v$ on each element $K$ is given by the following equation:

$$
\begin{aligned}
\int_{K} \nabla_{d} v \cdot \mathbf{q} d K= & -\int_{K} v_{0} \nabla \cdot \mathbf{q} d K \\
& +\int_{\partial K} v_{b} \mathbf{q} \cdot \mathbf{n} d s, \quad \forall \mathbf{q} \in V(K, j+1) .
\end{aligned}
$$

To investigate the approximation properties of the discrete weak spaces $S_{h}(j)$ and $\sum_{h}$, we use a local $L^{2}$ projection $Q_{h} u=\left\{Q_{0} u, Q_{b} u\right\}$ of $H^{1}(K)$ onto $P_{j}\left(K^{0}\right) \times P_{j+1}(\partial K)$ in this paper.

The discrete weak spaces $S_{h}(j)$ and $\sum_{h}$ need to possess some good approximation properties in order to provide an acceptable finite element scheme. In [17], the following two criteria were given as a general guideline for their construction.

(P1) For any $v \in S_{h}(j)$, if $\nabla_{d} v=0$ on $K$, then one must have $v \equiv$ constant on $K$. In other words, $v_{0}=v_{b} \equiv$ constant on $K$.

(P2) Let $u \in H_{0}^{1}(\Omega) \cap H^{m+1}(\Omega)$, where $0 \leq m \leq j+1$, be a smooth function on $\Omega$, and let $Q_{h} u=\left\{Q_{0} u, Q_{b} u\right\}$ be the $L^{2}$ projection/interpolation of $u$ in the corresponding finite element space $S_{h}(j)$ (recall that it is locally defined); then, the discrete weak gradient of $Q_{h} u$ provides a good approximation of $\nabla u$; that is, $\left\|\nabla_{d}\left(Q_{h} u\right)-\nabla u\right\| \leq C h^{m}\|u\|_{m+1}$ holds true.

Then, the characteristics weak Galerkin finite element method based on the weak Galerkin operator (16) and weak formulation (6) is to find $u_{h}(t)=\left\{u_{0}(t), u_{b}(t)\right\} \in S_{h}(j)$ for $0 \leq t \leq T$, satisfying $u_{b}(t)=Q_{b} \varphi$ on $\partial \Omega, 0<t \leq T$ and $u_{h}(0)=E_{h} u(0)\left(E_{h} u(0)\right.$ will be given in Section 3$)$ in $\Omega$ and the following equation:

$$
\begin{array}{r}
\left(\psi \frac{\partial u_{0}}{\partial \tau}, v_{0}\right)+a\left(u_{h}, v\right)=\left(f, v_{0}\right), \\
\forall v=\left\{v_{0}, v_{b}\right\} \in S_{h}^{0}(j), \quad 0<t \leq T,
\end{array}
$$

where $a(\cdot, \cdot)$ is the weak bilinear form defined by

$$
a(w, v)=\left(D \nabla_{d} w, \nabla_{d} v\right)=\int_{\Omega} D \nabla_{d} w \cdot \nabla_{d} v d x d y
$$

From the assumption of initial boundary value problem (1), we have

$$
\left(D \nabla_{d} w, \nabla_{d} w\right) \geq \alpha\left\|\nabla_{d} w\right\|^{2}
$$

We denote by $u_{h}^{i}=\left\{u_{0}^{i}, u_{b}^{i}\right\} \in S_{h}(j)(i=0,1, \ldots, N)$ the approximation of $u\left(t^{i}\right)(i=0,1, \ldots, N)$ and $\bar{u}_{0}^{i-1}=u_{0}(\bar{x}, \bar{y}$, $\left.t^{i-1}\right)(i=1,2, \ldots, N)$ the evaluation of $u_{0}$ at the point $\left(\bar{x}, \bar{y}, t^{i-1}\right)(i=1,2, \ldots, N)$. Then we obtain the characteristics weak Galerkin finite element scheme for the problem (1): find $u_{h}^{i}=\left\{u_{0}^{i}, u_{b}^{i}\right\} \in S_{h}(j)(i=0,1, \ldots, N)$, such that
$u_{h}^{0}=E_{h} u(0)\left(E_{h} u(0)\right.$ would be introduced in Section 3$)$ and, for $i=1,2, \ldots, N$,

$$
\begin{array}{r}
\left(a \frac{u_{0}^{i}-\bar{u}_{0}^{i-1}}{\Delta t}, v_{0}\right)+\left(D^{i} \nabla_{d} u_{h}^{i}, \nabla_{d} v\right)=\left(f\left(t^{i}\right), v_{0}\right), \\
\forall v=\left\{v_{0}, v_{b}\right\} \in S_{h}^{0}(j),
\end{array}
$$

where $D^{i}=D\left(t^{i}\right)$; that is,

$$
\begin{array}{r}
\left(a^{i} u_{0}^{i}, v_{0}\right)+\Delta t\left(D^{i} \nabla_{d} u_{h}^{i}, \nabla_{d} v\right)=\left(a^{i} \bar{u}_{0}^{i-1}+\Delta t f\left(t^{i}\right), v_{0}\right), \\
\forall v \in S_{h}^{0}(j) .
\end{array}
$$

It is obvious that (20) determines $u_{h}^{i}$ uniquely in terms of the $u_{h}^{0}$ data and $f$.

\section{A Weak Galerkin Elliptic Projection}

In the study of numerical methods for parabolic problems, an elliptic projection associated with the problem is usually introduced. The following Lemma 1, which is proved in Wang and Ye [17], gives the error estimate for the second order elliptic problem.

Lemma 1. Assume that $u \in L^{\infty}\left(0, T ; H^{m+1}(\Omega)\right)$ with $0 \leq m \leq$ $j+1$ and $\tilde{u}_{h}=\left(u_{h 0}, u_{h b}\right) \in S_{h}(j)$ are the solutions of the problems

$$
\begin{gathered}
-\nabla \cdot(D \nabla u)=F, \quad \text { in } \Omega, 0 \leq t \leq T, \\
u=g, \quad \text { on } \partial \Omega, 0 \leq t \leq T, \\
a\left(\tilde{u}_{h}, v\right)=\left(F, v_{0}\right), \quad \forall v=\left\{v_{0}, v_{b}\right\} \in S_{h}^{0}(j), 0 \leq t \leq T,
\end{gathered}
$$

respectively. Let $Q_{h} u=\left\{Q_{0} u, Q_{b} u\right\}$ be the $L^{2}$ projection of $u$ in the corresponding finite element space. Then there exists a positive constant $C$ independent of $h$ such that

$$
\begin{aligned}
& \left\|\nabla_{d}\left(Q_{h} u-\widetilde{u}_{h}\right)\right\|_{L^{\infty}\left(0, T ; L^{2}(\Omega)\right)} \\
& \quad \leq C\left(h^{2}\left\|F-Q_{0} F\right\|_{L^{\infty}\left(0, T ; L^{2}(\Omega)\right)}+h^{m}\|u\|_{L^{\infty}\left(0, T ; H^{m+1}(\Omega)\right)}\right), \\
& \left\|Q_{h} u-\widetilde{u}_{h}\right\|_{L^{\infty}\left(0, T ; L^{2}(\Omega)\right)} \\
& \quad \leq C\left(h^{2}\left\|F-Q_{0} F\right\|_{L^{\infty}\left(0, T ; L^{2}(\Omega)\right)}+h^{m+1}\|u\|_{L^{\infty}\left(0, T ; H^{m+1}(\Omega)\right)}\right),
\end{aligned}
$$

provided that the mesh-size $h$ is sufficiently small.

According to our problem (1), we introduce a weak Galerkin elliptic projection operator $E_{h}$ defined: find $E_{h} u=$ $\left(\widetilde{u}_{0}, \widetilde{u}_{b}\right) \in S_{h}(j)(0 \leq t \leq T)$, such that

$$
\begin{array}{r}
a\left(E_{h} u, v\right)=\left(-\nabla \cdot(D \nabla u), v_{0}\right), \\
\forall v=\left\{v_{0}, v_{b}\right\} \in S_{h}^{0}(j), \quad 0 \leq t \leq T .
\end{array}
$$


It has been proved in [17] that the solution $E_{h} u=\left(\tilde{u}_{0}, \tilde{u}_{b}\right) \epsilon$ $S_{h}(j)(0 \leq t \leq T)$ of $(24)$ is existence and uniqueness; then $E_{h} u(0)$ has been defined. So $u_{h}(0)$ or $u_{h}^{0}$ in (17) or (20) are well defined. $E_{h} u$.

From Lemma 1, we have the following error estimates for

Lemma 2. Assume that $u \in L^{\infty}\left(0, T ; H^{m+1}(\Omega)\right)$ with $0 \leq m \leq$ $j+1$ and $E_{h} u \in S_{h}(j)$ are the solutions of the problems (1) and (37), respectively. Let $Q_{h} u=\left\{Q_{0} u, Q_{b} u\right\}$ be the $L^{2}$ projection of $u$ in the corresponding finite element space. Then there exists a positive constant $C$ independent of $h$ such that

$$
\begin{aligned}
& \left\|\nabla_{d}\left(Q_{h} u-E_{h} u\right)\right\|_{L^{\infty}\left(0, T ; L^{2}(\Omega)\right)} \leq C h^{m}\|u\|_{L^{\infty}\left(0, T ; H^{m+1}(\Omega)\right)}, \\
& \left\|Q_{h} u-E_{h} u\right\|_{L^{\infty}\left(0, T ; L^{2}(\Omega)\right)} \leq C h^{m+1}\|u\|_{L^{\infty}\left(0, T ; H^{m+1}(\Omega)\right)},
\end{aligned}
$$

provided that the mesh-size $h$ is sufficiently small.

Differentiating (24) on $t$, we can prove the following Lemma 3 in the same way.

Lemma 3. Under the assumption of Lemma 2, if $u_{t} \in$ $L^{\infty}\left(0, T ; H^{m+1}(\Omega)\right)$, with $0 \leq m \leq j+1$, then there exists a positive constant $C$ independent of $h$ such that

$$
\begin{aligned}
& \left\|Q_{h} u_{t}-E_{h} u_{t}\right\|_{L^{\infty}\left(0, T ; L^{2}(\Omega)\right)} \\
& \quad \leq C h^{m+1}\left(\|u\|_{L^{\infty}\left(0, T ; H^{m+1}(\Omega)\right)}+\left\|u_{t}\right\|_{L^{\infty}\left(0, T ; H^{m+1}(\Omega)\right)}\right),
\end{aligned}
$$

provided that the mesh-size $h$ is sufficiently small.

\section{Optimal Order of Error Estimates in $L^{2}$ and the Discrete $H^{1}$}

In this section, we will develop the error estimates in the $H^{1}$ and $L^{2}$ norms for the characteristics weak Galerkin finite element method.

Assume that $u, u_{h}^{i}(i=0,1, \ldots, N)$ and $E_{h} u$ are the solutions of (1), (20), and (24), respectively. Let $Q_{h} u$ be the $L^{2}$ projection of $u$ in the corresponding finite element space, and

$$
\begin{gathered}
u_{h}^{i}-Q_{h} u^{i}=\xi^{i}+\eta^{i}, \\
\xi^{i}=u_{h}^{i}-E_{h} u^{i}\left(\xi_{0}^{i}=u_{0}^{i}-\widetilde{u}_{0}^{i}\right), \\
\eta^{i}=E_{h} u^{i}-Q_{h} u^{i}\left(\eta_{0}^{i}=\widetilde{u}_{0}^{i}-Q_{0} u^{i}\right), \quad i=1,2, \ldots, N .
\end{gathered}
$$

Then $\eta^{i}=E_{h} u^{i}-Q_{h} u^{i}$ can be handled by applying the results in Lemma 2. So, our main goal here is to bound $\xi^{i}=u_{h}^{i}-E_{h} u^{i}$.

Let $v=\left\{v_{0}, v_{b}\right\} \in S_{h}^{0}(j)$ be any test function. By testing (5) against the first component $v_{0}$, we arrive at the following:

$$
\left(\psi \frac{\partial\left(Q_{0} u\right)}{\partial \tau}, v_{0}\right)+\left(-\nabla \cdot(D \nabla u), v_{0}\right)=\left(f, v_{0}\right), \quad t \in(0, T]
$$

Subtracting (28) with $t=t^{i}$ from (20), and using (24) with $t=t^{i}$, we have the following error equation:

$$
\begin{aligned}
& \left(a^{i} \frac{\xi_{0}^{i}-\bar{\xi}_{0}^{i-1}}{\Delta t}, v_{0}\right)+\left(D^{i} \nabla_{d} \xi^{i}, \nabla_{d} v\right) \\
& =\left(\psi^{i} \frac{\partial\left(Q_{0} u^{i}\right)}{\partial \tau}-a^{i} \frac{Q_{0} u^{i}-Q_{0} \bar{u}^{i-1}}{\Delta t}, v_{0}\right) \\
& -\left(a^{i} \frac{\eta_{0}^{i}-\bar{\eta}_{0}^{i-1}}{\Delta t}, v_{0}\right), \quad i=1,2, \ldots, N,
\end{aligned}
$$

and $\xi^{0}=\left\{\xi_{0}^{0}, \xi_{b}^{0}\right\}=\{0,0\}$.

In order to derive error estimates, we give three lemmas which have been proved in [19].

Lemma 4. If $\eta \in L^{2}(\Omega)$ and $\bar{\eta}(x, y)=\eta\left(x-g_{1}(x, y) \Delta t, y-\right.$ $\left.g_{2}(x, y) \Delta t\right)$, where $g_{1}, g_{2}, g_{1}^{\prime}$, and $g_{2}^{\prime}$ are bounded, then

$$
\begin{aligned}
\|\eta-\bar{\eta}\|_{-1} & \leq C\|\eta\| \Delta t, \\
\|\eta-\bar{\eta}\| & \leq C\|\eta\|_{1} \Delta t .
\end{aligned}
$$

Lemma 5. Let $F(\mathbf{x})=\mathbf{x}-G(\mathbf{x}) \Delta t$, where $\mathbf{x}=(x, y), G(\mathbf{x})=$ $\left(g_{1}(\mathbf{x}), g_{2}(\mathbf{x})\right)$; then for all $\varphi \in W^{1,2}(\Omega)$,

$$
\begin{gathered}
\|\varphi \circ F\| \leq\|\varphi\|^{2}(1+\gamma C \Delta t), \\
\left\|\varphi \circ F^{-1}\right\| \leq\|\varphi\|^{2}(1+\gamma C \Delta t), \\
|\gamma| \leq 1 .
\end{gathered}
$$

Lemma 6. Let $Q_{h} u=\left\{Q_{0} u, Q_{b} u\right\}$ be the $L^{2}$ projection of $u$ in the corresponding finite element space; then

$$
\begin{gathered}
\left\|\psi^{i} \frac{\partial\left(Q_{0} u^{i}\right)}{\partial \tau}-a^{i} \frac{Q_{0} u^{i}-Q_{0} \bar{u}^{i-1}}{\Delta t}\right\|^{2} \\
\leq 2 \Delta t\left\|\frac{\varphi^{4}}{a^{2}}\right\|_{0, \infty}\left\|\frac{\partial^{2} u}{\partial \tau^{2}}\right\|_{L^{2}\left(\Omega \times\left[t^{i-1}, t^{i}\right]\right)}^{2} .
\end{gathered}
$$

The error estimates for the characteristics weak Galerkin finite element method in $L^{2}$ and $H^{1}$ norms are provided in the next two theorems.

Theorem 7. Assume that $u, u_{h}^{i}(i=0,1, \ldots, N)$, and $E_{h} u$ are the solutions of (1), (20), and (24), respectively. If $u, u_{t} \in$ $L^{\infty}\left(0, T ; H^{m+1}(\Omega)\right)$ and $u_{t t} \in L^{2}\left(0, T ; L^{2}(\Omega)\right)$, with $0 \leq m \leq$ $j+1$, then there exists a positive constant $C$ independent of $h$ and $\Delta t$ such that

$$
\begin{aligned}
\max _{0 \leq i \leq N} & \left\|u_{h}^{i}-Q_{h} u^{i}\right\| \\
\leq & C \Delta t\left\|\frac{\partial^{2} u}{\partial \tau^{2}}\right\|_{L^{2}\left(0, T ; L^{2}(\Omega)\right)} \\
& +C h^{m+1}\left(\|u\|_{L^{\infty}\left(0, T ; H^{m+1}(\Omega)\right)}^{2}+\left\|\frac{\partial u}{\partial t}\right\|_{L^{\infty}\left(0, T ; H^{m+1}(\Omega)\right)}^{2}\right)^{1 / 2}
\end{aligned}
$$

provided that the mesh-size $h$ and $\Delta t$ are sufficiently small. 
Proof. Taking $v=\xi^{i}$ in (29), we arrive at

$$
\begin{aligned}
\left(a^{i} \frac{\xi_{0}^{i}-\bar{\xi}_{0}^{i-1}}{\Delta t}, \xi_{0}^{i}\right)+\left(D^{i} \nabla_{d} \xi^{i}, \nabla_{d} \xi^{i}\right) \\
=\left(\psi^{i} \frac{\partial\left(Q_{0} u^{i}\right)}{\partial \tau}-a^{i} \frac{Q_{0} u^{i}-Q_{0} \bar{u}^{i-1}}{\Delta t}, \xi_{0}^{i}\right) \\
-\left(a^{i} \frac{\eta_{0}^{i}-\bar{\eta}_{0}^{i-1}}{\Delta t}, \xi_{0}^{i}\right), \quad i=1,2, \ldots, N .
\end{aligned}
$$

For $i=1,2, \ldots, N$, we first estimate the left items of (34) as follows:

$$
\begin{aligned}
& \left(a^{i} \frac{\xi_{0}^{i}-\bar{\xi}_{0}^{i-1}}{\Delta t}, \xi_{0}\right)+\left(D^{i} \nabla_{d} \xi^{i}, \nabla_{d} \xi^{i}\right) \\
& =\frac{1}{2 \Delta t}\left[\left(a^{i} \xi_{0}^{i}, \xi_{0}^{i}\right)-\left(a^{i} \bar{\xi}_{0}^{i-1}, \bar{\xi}_{0}^{i-1}\right)+\left(a^{i} \xi_{0}^{i}-\bar{\xi}_{0}^{i-1}, \xi_{0}^{i}-\bar{\xi}_{0}^{i-1}\right)\right] \\
& \quad+\left(D^{i} \nabla_{d} \xi^{i}, \nabla_{d} \xi^{i}\right) \\
& \geq \frac{1}{2 \Delta t}\left[\left(a^{i} \xi_{0}^{i}, \xi_{0}^{i}\right)-\left(a^{i} \bar{\xi}_{0}^{i-1}, \bar{\xi}_{0}^{i-1}\right)\right]+\alpha\left\|\nabla_{d} \xi^{i}\right\|^{2} \\
& =\frac{1}{2 \Delta t}\left[\left(a^{i} \xi_{0}^{i}, \xi_{0}^{i}\right)-\left(a^{i} \xi_{0}^{i-1}, \xi_{0}^{i-1}\right)\left(1+\gamma^{i} C \Delta t\right)\right] \\
& \quad+\alpha\left\|\nabla_{d} \xi^{i}\right\|^{2}, \quad\left|\gamma^{i}\right| \leq 1,
\end{aligned}
$$

where the last step uses Lemma 5.

Next, we estimate the right items of (34). Using Lemma 6, we have that

$$
\begin{gathered}
\left(\psi^{i} \frac{\partial\left(Q_{0} u^{i}\right)}{\partial \tau}-a^{i} \frac{Q_{0} u^{i}-Q_{0} \bar{u}^{i-1}}{\Delta t}, \xi_{0}^{i}\right) \\
\leq C \Delta t\left\|\frac{\partial^{2} u}{\partial \tau^{2}}\right\|_{L^{2}\left(\Omega \times\left[t^{i-1}, t^{i}\right]\right)}^{2}+\left\|\xi_{0}^{i}\right\|^{2} .
\end{gathered}
$$

Write $\eta_{0}^{i}-\bar{\eta}_{0}^{i-1}$ as the sum $\left(\eta_{0}^{i}-\eta_{0}^{i-1}\right)+\left(\eta_{0}^{i-1}-\bar{\eta}_{0}^{i-1}\right)$ and use $\left\|\xi_{0}^{i}\right\|_{1} \leq C\left|\xi_{0}^{i}\right|_{1}$, for all $\xi_{0}^{i} \in S_{h}^{0}(j)$; then

$$
\begin{aligned}
\left(a \frac{\eta_{0}^{i}-\eta_{0}^{i-1}}{\Delta t}, \xi_{0}^{i}\right) & \leq \frac{C}{\Delta t} \int_{t^{i-1}}^{t^{i}}\left\|\frac{\partial \eta_{0}}{\partial t}\right\|_{-1} d t\left\|\xi_{0}^{i}\right\|_{1} \\
& \leq C\left\|\frac{\partial \eta_{0}}{\partial t}\right\|_{L^{\infty}\left(t^{i-1}, t^{i} ; W^{-1,2}(\Omega)\right)}^{2}+\varepsilon\left\|\xi_{0}^{i}\right\|_{1}^{2} \\
& \leq C\left\|\frac{\partial \eta_{0}}{\partial t}\right\|_{L^{\infty}\left(t^{i-1}, t^{i} ; L^{2}(\Omega)\right)}^{2}+\varepsilon\left\|\xi_{0}^{i}\right\|_{1}^{2}
\end{aligned}
$$

And using Lemma 4, we have

$$
\begin{aligned}
\left(a \frac{\eta_{0}^{i-1}-\bar{\eta}_{0}^{i-1}}{\Delta t}, \xi_{0}^{i}\right) & \leq C\left\|\frac{\eta_{0}^{i-1}-\bar{\eta}_{0}^{i-1}}{\Delta t}\right\|_{-1}\left\|\xi_{0}^{i}\right\|_{1} \\
& \leq C\left\|\eta_{0}^{i-1}\right\|^{2}+\varepsilon\left\|\xi_{0}^{i}\right\|_{1}^{2} .
\end{aligned}
$$

This completes the treatment of the right-hand side.

The inequalities (35), (36), (37), and (38) can be combined with (34) to give the recursion relation

$$
\begin{aligned}
& \frac{1}{2 \Delta t}\left[\left(a^{i} \xi_{0}^{i}, \xi_{0}^{i}\right)-\left(a^{i} \xi_{0}^{i-1}, \xi_{0}^{i-1}\right)\right]+\frac{\alpha}{2}\left\|\nabla_{d} \xi^{i}\right\|^{2} \\
& \leq \frac{\gamma^{i} C}{2}\left(a^{i} \xi_{0}^{i-1}, \xi_{0}^{i-1}\right)+C \Delta t\left\|\frac{\partial^{2} u}{\partial \tau^{2}}\right\|_{L^{2}\left(t^{i-1}, t^{i} ; L^{2}(\Omega)\right)}^{2} \\
& \quad+C\left\|\frac{\partial \eta_{0}}{\partial t}\right\|_{L^{\infty}\left(t^{i-1}, t^{i} ; L^{2}(\Omega)\right)}^{2}+C\left\|\eta_{0}^{i-1}\right\|^{2}+\left\|\xi_{0}^{i}\right\|^{2} .
\end{aligned}
$$

If (39) is multiplied by $2 \Delta t$ and summed in time and if it is noted that $\xi_{0}^{0}=0$, then it follows that

$$
\begin{aligned}
\left\|\xi_{0}^{i}\right\|^{2}+\alpha \Delta t \sum_{j=1}^{i}\left\|\nabla_{d} \xi^{j}\right\|^{2} \leq & C(\Delta t)^{2}\left\|\frac{\partial^{2} u}{\partial \tau^{2}}\right\|_{L^{2}\left(0, T ; L^{2}(\Omega)\right)}^{2} \\
& +C\left\|\frac{\partial \eta_{0}}{\partial t}\right\|_{L^{\infty}\left(0, T ; L^{2}(\Omega)\right)}^{2} \\
& +C\left\|\eta_{0}\right\|_{L^{\infty}\left(0, T ; L^{2}(\Omega)\right)}^{2} \\
& +2 C \Delta t \sum_{j=1}^{i}\left\|\xi_{0}^{j}\right\|^{2}
\end{aligned}
$$

Using the discrete Gronwall inequality and Lemmas 2 and 3, when $\Delta t$ is sufficiently small, we have

$$
\begin{aligned}
\max _{0 \leq i \leq N}\left\|\xi_{0}^{i}\right\|^{2} & \\
\leq & C(\Delta t)^{2}\left\|\frac{\partial^{2} u}{\partial \tau^{2}}\right\|_{L^{2}\left(0, T ; L^{2}(\Omega)\right)}^{2} \\
& +C h^{2 m+2}\left(\|u\|_{L^{\infty}\left(0, T ; H^{m+1}(\Omega)\right)}^{2}+\left\|\frac{\partial u}{\partial t}\right\|_{L^{\infty}\left(0, T ; H^{m+1}(\Omega)\right)}^{2}\right) .
\end{aligned}
$$

Then, the result of Theorem 7 follows from (41) and $\left\|\xi^{i}\right\|=$ $\left\|\xi_{0}^{i}\right\|$. The proof is complete.

Theorem 8. Assume that $u, u_{h}^{i}(i=0,1, \ldots, N)$, and $E_{h} u$ are the solutions of (1), (20), and (24), respectively. If $u, u_{t} \in L^{\infty}\left(0, T ; H^{m+1}(\Omega)\right)$ and $u_{t t} \in L^{2}\left(0, T ; L^{2}(\Omega)\right)$ with 
$0 \leq m \leq j+1$, then there exists a positive constant $C$ independent of $h$ and $\Delta t$ such that

$$
\begin{aligned}
\max _{0 \leq i \leq N} & \left\|\nabla_{d}\left(u_{h}^{i}-Q_{h} u^{i}\right)\right\| \\
\leq & C \Delta t\left\|\frac{\partial^{2} u}{\partial \tau^{2}}\right\|_{L^{2}\left(0, T ; L^{2}(\Omega)\right)} \\
& +C h^{m}\left(\|u\|_{L^{\infty}\left(0, T ; H^{m+1}(\Omega)\right)}^{2}+\left\|\frac{\partial u}{\partial t}\right\|_{L^{\infty}\left(0, T ; H^{m+1}(\Omega)\right)}^{2}\right)^{1 / 2},
\end{aligned}
$$

provided that the mesh-size $h$ and $\Delta t$ are sufficiently small.

Proof. For $i=1,2, \ldots, N$, taking $v=\left(\xi^{i}-\xi^{i-1}\right) / \Delta t$ in (29), we arrive at the following:

$$
\begin{array}{r}
\left(a^{i} \frac{\xi_{0}^{i}-\bar{\xi}_{0}^{i-1}}{\Delta t}, \frac{\xi_{0}^{i}-\xi_{0}^{i-1}}{\Delta t}\right)+\left(D^{i} \nabla_{d} \xi^{i}, \nabla_{d} \frac{\xi^{i}-\xi^{i-1}}{\Delta t}\right) \\
=\left(\psi^{i} \frac{\partial\left(Q_{0} u^{i}\right)}{\partial \tau}-a^{i} \frac{Q_{0} u^{i}-Q_{0} \bar{u}^{i-1}}{\Delta t}, \frac{\xi_{0}^{i}-\xi_{0}^{i-1}}{\Delta t}\right) \\
\quad-\left(a^{i} \frac{\eta_{0}^{i}-\bar{\eta}_{0}^{i-1}}{\Delta t}, \frac{\xi_{0}^{i}-\xi_{0}^{i-1}}{\Delta t}\right), \quad i=1,2, \ldots, N .
\end{array}
$$

By Lemma 4, the left-hand side satisfies the inequality

$$
\begin{aligned}
( & \left.a^{i} \frac{\xi_{0}^{i}-\bar{\xi}_{0}^{i-1}}{\Delta t}, \frac{\xi_{0}^{i}-\xi_{0}^{i-1}}{\Delta t}\right)+\left(D^{i} \nabla_{d} \xi^{i}, \nabla_{d} \frac{\xi^{i}-\xi^{i-1}}{\Delta t}\right) \\
= & \left(a^{i} \frac{\xi_{0}^{i}-\xi_{0}^{i-1}}{\Delta t}, \frac{\xi_{0}^{i}-\xi_{0}^{i-1}}{\Delta t}\right) \\
& -\left(a^{i} \frac{\bar{\xi}_{0}^{i-1}-\xi_{0}^{i-1}}{\Delta t}, \frac{\xi_{0}^{i}-\xi_{0}^{i-1}}{\Delta t}\right) \\
& +\frac{1}{2 \Delta t}\left[\left(D^{i} \nabla_{d} \xi^{i}, \nabla_{d} \xi^{i}\right)-\left(D^{i} \nabla_{d} \xi^{i-1}, \nabla_{d} \xi^{i-1}\right)\right] \\
\geq & \left(a^{i} \frac{\xi_{0}^{i}-\xi_{0}^{i-1}}{\Delta t}, \frac{\xi_{0}^{i}-\xi_{0}^{i-1}}{\Delta t}\right)-C\left\|\xi_{0}^{i-1}\right\|_{1}^{2} \\
& -\varepsilon\left\|\frac{\xi_{0}^{i}-\xi_{0}^{i-1}}{\Delta t}\right\| \|^{2} \\
& +\frac{1}{2 \Delta t}\left[\left(D^{i} \nabla_{d} \xi^{i}, \nabla_{d} \xi^{i}\right)-\left(D^{i} \nabla_{d} \xi^{i-1}, \nabla_{d} \xi^{i-1}\right)\right] .
\end{aligned}
$$

The right-hand side terms can be estimated as below. First

$$
\begin{gathered}
\left(\psi^{i} \frac{\partial\left(Q_{0} u^{i}\right)}{\partial \tau}-a^{i} \frac{Q_{0} u^{i}-Q_{0} \bar{u}^{i-1}}{\Delta t}, \frac{\xi_{0}^{i}-\xi_{0}^{i-1}}{\Delta t}\right) \\
\leq C \Delta t\left\|\frac{\partial^{2} u}{\partial \tau^{2}}\right\|_{L^{2}\left(t^{i-1}, t^{i} ; L^{2}(\Omega)\right)}^{2}+\varepsilon\left\|\frac{\xi_{0}^{i}-\xi_{0}^{i-1}}{\Delta t}\right\|^{2} .
\end{gathered}
$$

Second,

$$
\begin{aligned}
\left(a^{i} \frac{\eta_{0}^{i}-\bar{\eta}_{0}^{i-1}}{\Delta t}, \frac{\xi_{0}^{i}-\xi_{0}^{i-1}}{\Delta t}\right) \leq & C\left\|\frac{\partial \eta_{0}}{\partial t}\right\|_{L^{\infty}\left(t^{i-1}, t^{i} L^{2}(\Omega)\right)}^{2} \\
& +C\left\|\eta_{0}^{i-1}\right\|_{1}^{2}+\varepsilon\left\|\frac{\xi_{0}^{i}-\xi_{0}^{i-1}}{\Delta t}\right\|^{2} .
\end{aligned}
$$

Combining (44), (45), and (46) with (43), we arrive at the following:

$$
\begin{aligned}
& \frac{1}{2 \Delta t}\left[\left(D^{i} \nabla_{d} \xi^{i}, \nabla_{d} \xi^{i}\right)-\left(D^{i} \nabla_{d} \xi^{i-1}, \nabla_{d} \xi^{i-1}\right)\right] \\
& \leq C \Delta t\left\|\frac{\partial^{2} u}{\partial \tau^{2}}\right\|_{L^{2}\left(t^{i-1}, t^{i} ; L^{2}(\Omega)\right)}^{2} \\
& +C\left\|\frac{\partial \eta_{0}}{\partial t}\right\|_{L^{\infty}\left(t^{i-1}, t^{i} ; L^{2}(\Omega)\right)}^{2} \\
& +C\left\|\eta_{0}^{i-1}\right\|_{1}^{2}+C\left\|\xi_{0}^{i-1}\right\|_{1}^{2} .
\end{aligned}
$$

Multiplying $2 \Delta t$ and then summing over $i$ from 1 to $i$ at both sides of (47) and noting that $\xi^{0}=\{0,0\}$, we obtain

$$
\begin{aligned}
\left\|\nabla_{d} \xi^{i}\right\|^{2} \leq & C(\Delta t)^{2}\left\|\frac{\partial^{2} u}{\partial \tau^{2}}\right\|_{L^{2}\left(0, T ; L^{2}(\Omega)\right)}^{2} \\
& +C\left\|\frac{\partial \eta_{0}}{\partial t}\right\|_{L^{\infty}\left(0, T ; L^{2}(\Omega)\right)}^{2} \\
& +C\left\|\eta_{0}\right\|_{L^{\infty}\left(0, T ; H^{1}(\Omega)\right)}^{2} \\
& +C \Delta t \sum_{j=2}^{i}\left\|\xi_{0}^{j-1}\right\|_{1}^{2}, \quad i=1,2, \ldots, N .
\end{aligned}
$$

By $\left\|\xi_{0}^{i}\right\|_{1} \leq C\left|\xi_{0}^{i}\right|_{1}$, for all $\xi_{0}^{i} \in S_{h}^{0}(j)$ and the discrete Gronwall inequality and Lemmas 2 and 3 , when $\Delta t$ is sufficiently small, we have

$$
\begin{array}{r}
\max _{0 \leq i \leq N}\left\|\nabla_{d} \xi^{i}\right\|^{2} \\
\leq C(\Delta t)^{2}\left\|\frac{\partial^{2} u}{\partial \tau^{2}}\right\|_{L^{2}\left(0, T ; L^{2}(\Omega)\right)}^{2} \\
+C h^{2 m+2}\left(\|u\|_{L^{\infty}\left(0, T ; H^{m+1(\Omega)}\right)}^{2 m}+\left\|\frac{\partial u}{\partial t}\right\|_{L^{\infty}\left(0, T ; H^{m+1}(\Omega)\right)}^{2}\right), \\
i=1,2, \ldots, N .
\end{array}
$$


Using Lemma 2, (49), and triangle inequality, we have

$$
\begin{aligned}
& \max _{0 \leq i \leq N}\left\|\nabla_{d}\left(u_{h}^{i}-Q_{h} u^{i}\right)\right\|^{2} \\
& \leq C(\Delta t)^{2}\left\|\frac{\partial^{2} u}{\partial \tau^{2}}\right\|_{L^{2}\left(0, T ; L^{2}(\Omega)\right)}^{2} \\
& +C h^{2 m+2}\left(\|u\|_{L^{\infty}\left(0, T ; H^{m+1(\Omega)}\right)}^{2}+\left\|\frac{\partial u}{\partial t}\right\|_{L^{\infty}\left(0, T ; H^{m+1}(\Omega)\right)}^{2}\right), \\
& i=1,2, \ldots, N .
\end{aligned}
$$

This completes the proof.

\section{Numerical Experiments}

In this section, we present two examples to demonstrate the convergence order of the studied characteristics weak Galerkin finite element method. Let $\mathscr{T}_{h}$ be a quasi-uniform triangulation with mesh-size $h$ and let $\Delta t=T / N$ be the time step. In the numerical tests, discrete weak spaces $S_{h}^{0}(0)$ and $\Sigma_{h}$, with $V(K, 1)$ being the lowest order Raviart-Thomas element $\mathrm{RT}_{0}(K)$ on the triangle $K$, are considered. We denote the numerical solution of $u(x, y, T)$ by $u_{h}^{N}$ and the error by $e_{h}=\left\{e_{0}, e_{b}\right\}=u_{h}^{N}-Q_{h} u(x, y, T)$.

Example 1. In this example, we take $\Omega=(0,1) \times(0,1)$, $T=1$, the coefficient matrix $D=\left[\begin{array}{cc}x^{2}+y^{2}+1 & x y \\ x y & x^{2}+y^{2}+1\end{array}\right]$, the exact solution $u=\cos \left(2 \pi t^{2}\right) \sin (\pi x) \sin (\pi y)$ which satisfies homogeneous Dirichlet boundary condition, and the initial condition $u_{0}(x, y)=\sin (\pi x) \sin (\pi y)$. Here are two settings of $B$ : (i) $B=-[\cos (\pi x) \sin (\pi y), \sin (\pi x) \cos (\pi y)]^{T}$ and (ii) $B=$ $[x(1-x), y(1-y)]^{T}$. With each selection of $B$, the source term $f$ can be obtained by substituting the exact solution into the equation. For a fixed mesh ratio $\Delta t / h=0.5$, the norm of $e_{0}$ and $\nabla_{d} e_{h}$ with the options (i) and (ii) of $B$ is reported in Tables 1 and 2, respectively. It is observed that the numerical results reflect the convergence order of scheme (20) and support our theoretical conclusions in Section 4 .

Example 2. In this example, we study the case for which the exact solution has a sharp front moving with time. The exact solution is given by $u(x, y, t)=\widetilde{u}(x+x(1-x) t, y+y(1-y) t)$, where

$$
\widetilde{u}(x, y)=1-\frac{1}{1+e^{-\left((x-1)^{2}+(y-1)^{2}-r^{2}\right) / c}}
$$

$r=0.5$, and $c=0.02$. We set $\Omega=(0,1) \times(0,1), T=1$, the coefficient matrix $D=\left[\begin{array}{ll}1 & 0 \\ 0 & 1\end{array}\right]$, and $B=-[x(1-x), y(1-y)]^{T}$. The norm of $e_{0}$ and $\nabla_{d} e_{h}$ for $\Delta t / h=0.5$ is reported in Table 3. It shows that the characteristics weak Galerkin finite element method can simulate the solution with a sharp front effectively.
TABLE 1: Error behavior of Example 1 with the setting (i) of $B$ and $\Delta t / h=0.5$.

\begin{tabular}{cccccc}
\hline$\Delta t$ & $h$ & $\left\|e_{0}\right\|$ & Order $\approx$ & $\left\|\nabla_{d} e\right\|$ & Order $\approx$ \\
\hline $2^{-4}$ & $2^{-3}$ & $6.649 e-2$ & - & $3.324 e-1$ & - \\
$2^{-5}$ & $2^{-4}$ & $3.537 e-2$ & 0.911 & $1.819 e-1$ & 0.869 \\
$2^{-6}$ & $2^{-5}$ & $1.885 e-2$ & 0.908 & $9.520 e-2$ & 0.934 \\
$2^{-7}$ & $2^{-6}$ & $9.473 e-3$ & 0.993 & $4.767 e-2$ & 0.998 \\
$2^{-8}$ & $2^{-7}$ & $4.710 e-3$ & 1.008 & $2.369 e-2$ & 1.008 \\
\hline
\end{tabular}

TABLE 2: Error behavior of Example 1 with the setting (ii) of $B$ and $\Delta t / h=0.5$.

\begin{tabular}{cccccc}
\hline$\Delta t$ & $h$ & $\left\|e_{0}\right\|$ & Order $\approx$ & $\left\|\nabla_{d} e\right\|$ & Order \\
\hline $2^{-4}$ & $2^{-3}$ & $4.833 e-2$ & - & $2.808 e-1$ & - \\
$2^{-5}$ & $2^{-4}$ & $2.761 e-2$ & 0.807 & $1.515 e-1$ & 0.890 \\
$2^{-6}$ & $2^{-5}$ & $1.477 e-2$ & 0.902 & $7.901 e-2$ & 0.939 \\
$2^{-7}$ & $2^{-6}$ & $7.665 e-3$ & 0.946 & $4.050 e-2$ & 0.964 \\
$2^{-8}$ & $2^{-7}$ & $3.916 e-3$ & 0.968 & $2.060 e-2$ & 0.975 \\
\hline
\end{tabular}

TABLE 3: Error behavior of Example 2 for $\Delta t / h=0.5$.

\begin{tabular}{lccccc}
\hline$\Delta t$ & $h$ & $\left\|e_{0}\right\|$ & Order $\approx$ & $\left\|\nabla_{d} e\right\|$ & Order $\approx$ \\
\hline $2^{-5}$ & $2^{-4}$ & $3.941 e-2$ & - & $7.965 e-1$ & - \\
$2^{-6}$ & $2^{-5}$ & $1.942 e-2$ & 1.021 & $3.352 e-1$ & 1.248 \\
$2^{-7}$ & $2^{-6}$ & $9.733 e-3$ & 0.996 & $1.562 e-1$ & 1.102 \\
$2^{-8}$ & $2^{-7}$ & $4.934 e-3$ & 0.980 & $7.668 e-2$ & 1.025 \\
$2^{-9}$ & $2^{-8}$ & $2.502 e-3$ & 0.980 & $3.773 e-2$ & 1.023 \\
\hline
\end{tabular}

\section{Conflict of Interests}

The authors declare that there is no conflict of interests regarding the publication of this paper.

\section{Acknowledgments}

The project is supported by the National Natural Science Fund (11171193), the Fund of the Natural Science of Shandong Province (ZR2011MA016), and a Project of Shandong Province Science and Technology Development Program (2012GGB01198).

\section{References}

[1] B. R. Baliga and S. V. Patankar, "A new finite-element formulation for convection-diffusion problems," Numerical Heat Transfer, vol. 3, no. 4, pp. 393-409, 1980.

[2] K. Eriksson and C. Johnson, "Adaptive streamline diffusion finite element methods for stationary convection-diffusion problems," Mathematics of Computation, vol. 60, no. 201, pp. 167-188, 1993.

[3] R. Codina, "Comparison of some finite element methods for solving the diffusion-convection-reaction equation," Computer Methods in Applied Mechanics and Engineering, vol. 156, no. 1-4, pp. 185-210, 1998. 
[4] V. John, J. M. Maubach, and L. Tobiska, "Nonconforming streamline-diffusion-finite-elementmethods for convectiondiffusion problems," Numerische Mathematik, vol. 78, no. 2, pp. 165-188, 1997.

[5] L. P. Franca, A. Nesliturk, and M. Stynes, "On the stability of residual-free bubbles for convection-diffusion problems and their approximation by a two-level finite element method," Computer Methods in Applied Mechanics and Engineering, vol. 166, no. 1-2, pp. 35-49, 1998.

[6] R. Codina, "On stabilized finite element methods for linear systems of convection-diffusion-reaction equations," Computer Methods in Applied Mechanics and Engineering, vol. 188, no. 1-3, pp. 61-82, 2000.

[7] T. E. Tezduyar and Y. J. Park, "Discontinuity-capturing finite element formulations for nonlinear convection-diffusion-reaction equations," Computer Methods in Applied Mechanics and Engineering, vol. 59, no. 3, pp. 307-325, 1986.

[8] R. Codina, "A discontinuity-capturing crosswind-dissipation for the finite element solution of the convection-diffusion equation," Computer Methods in Applied Mechanics and Engineering, vol. 110, no. 3-4, pp. 325-342, 1993.

[9] A. C. Galeão, R. C. Almeida, S. M. C. Malta, and A. F. D. Loula, "Finite element analysis of convection dominated reactiondiffusion problems," Applied Numerical Mathematics, vol. 48, no. 2, pp. 205-222, 2004.

[10] J. Xu and L. Zikatanov, "A monotone finite element scheme for convection-diffusion equations," Mathematics of Computation, vol. 68, no. 228, pp. 1429-1446, 1999.

[11] H. Rui and M. Tabata, "A second order characteristic finite element scheme for convection-diffusion problems," Numerische Mathematik, vol. 92, no. 1, pp. 161-177, 2002.

[12] G. E. Schneider and M. J. Raw, "A skewed, positive influence coefficient upwinding procedure for control-volume-based finite-element convection-diffusion computation," Numerical Heat Transfer, vol. 9, no. 1, pp. 1-26, 1986.

[13] R. D. Lazarov, I. D. Mishev, and P. S. Vassilevski, "Finite volume methods for convection-diffusion problems," SIAM Journal on Numerical Analysis, vol. 33, no. 1, pp. 31-55, 1996.

[14] S.-H. Chou, D. Y. Kwak, and P. S. Vassilevski, "Mixed upwinding covolume methods on rectangular grids for convectiondiffusion problems," SIAM Journal on Scientific Computing, vol. 21, no. 1, pp. 145-165, 1999.

[15] O. Axelsson and W. Layton, "Defect correction methods for convection dominated convectiondiffusion problems," ESAIM: Mathematical Modelling and Numerical Analysis, vol. 24, no. 4, pp. 423-455, 1990.

[16] B. Cockburn and C.-W. Shu, "The local discontinuous Galerkin method for time-dependent convection-diffusion systems," SIAM Journal on Numerical Analysis, vol. 35, no. 6, pp. 24402463, 1998.

[17] J. Wang and X. Ye, "A weak Galerkin finite element method for second-order elliptic problems," Journal of Computational and Applied Mathematics, vol. 241, pp. 103-115, 2013.

[18] L. Mu, J. Wang, Y. Wang, and X. Ye, "A computational study of the weak Galerkin method for second-order elliptic equations," Numerical Algorithms, vol. 63, no. 4, pp. 753-777, 2013.

[19] J. J. Douglas and T. F. Russell, "Numerical methods for convection-dominated diffusion problems based on combining the method of characteristics with finite element or finite difference procedures," SIAM Journal on Numerical Analysis, vol. 19, no. 5, pp. 871-885, 1982. 


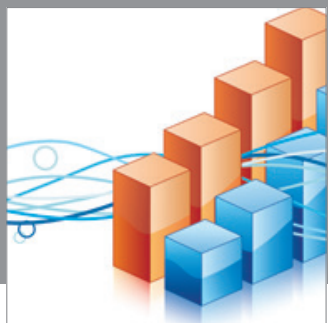

Advances in

Operations Research

mansans

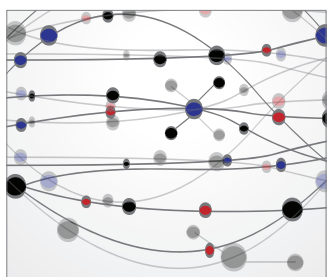

The Scientific World Journal
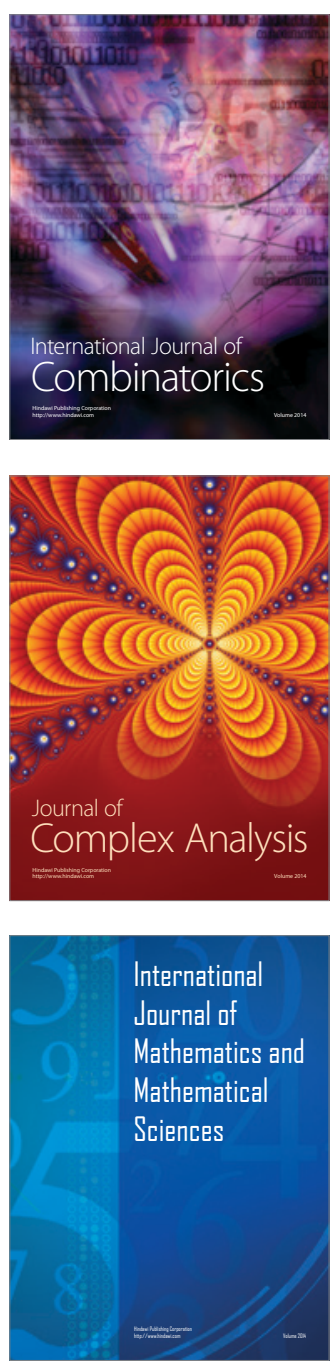
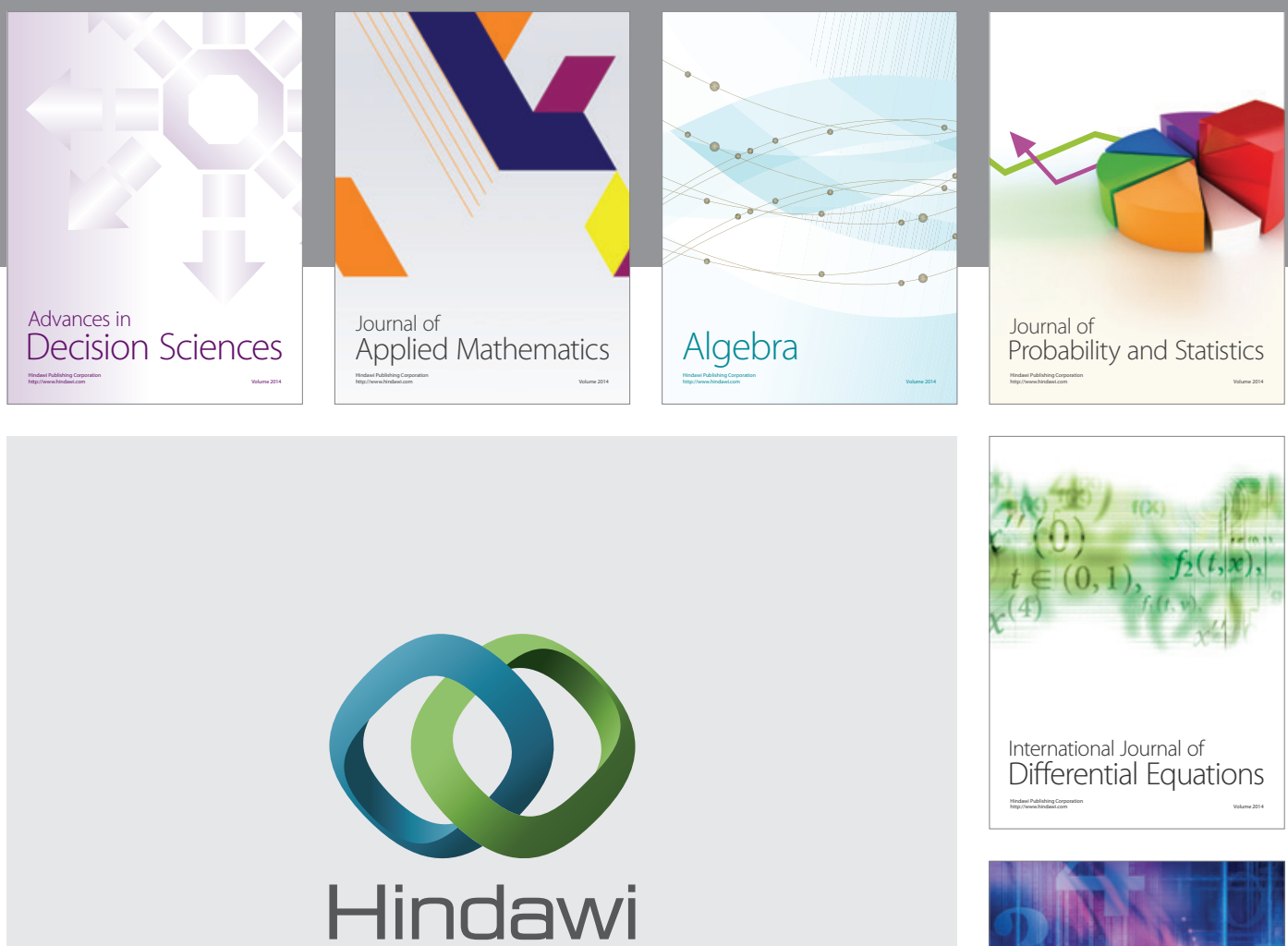

Submit your manuscripts at http://www.hindawi.com
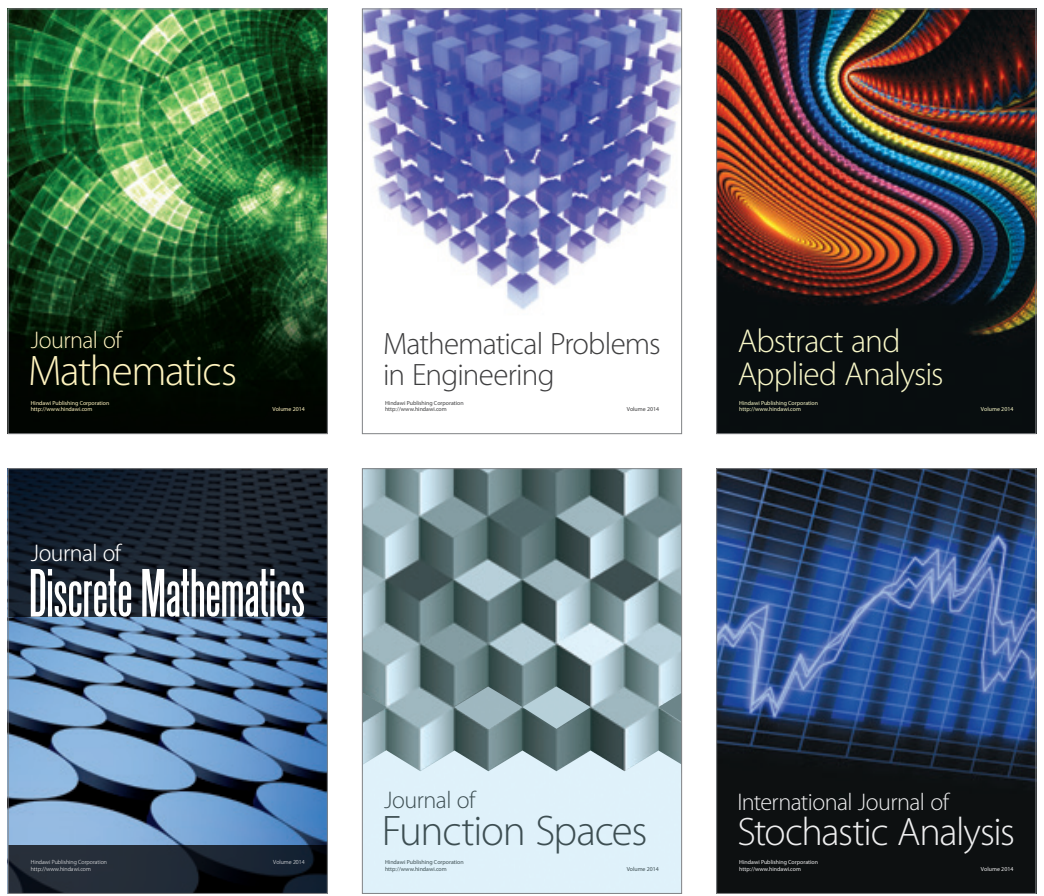

Journal of

Function Spaces

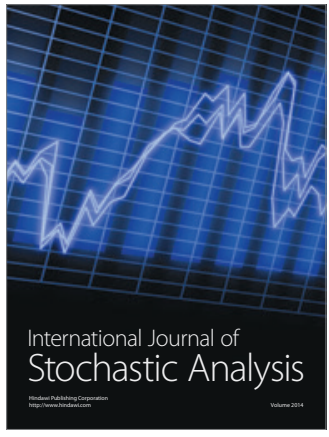

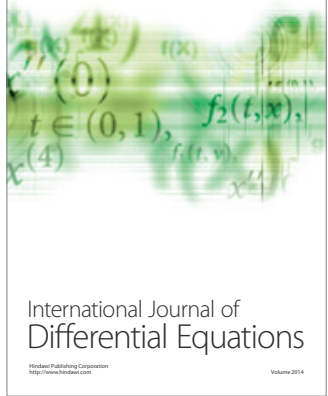
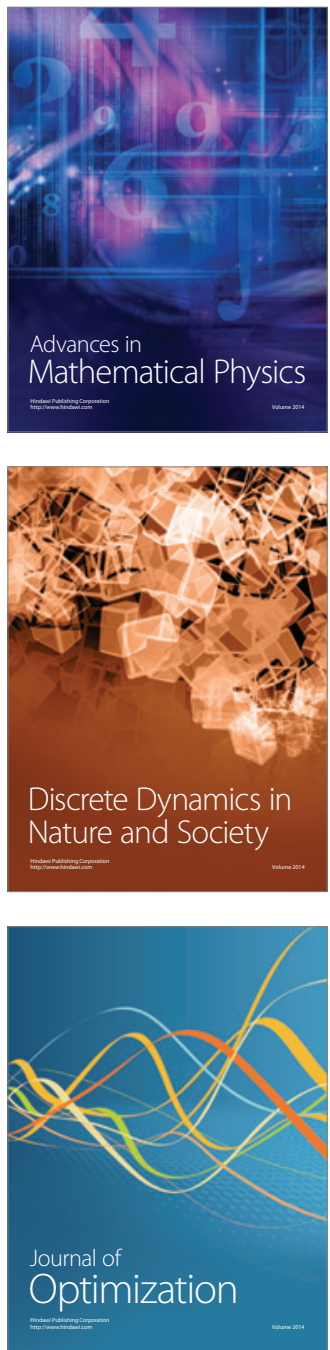